\title{
The Hedgehog Signaling Pathway: A Promising Target for Therapeutic Development
}

\author{
* Nahla AM Hamed \\ Faculty of Medicine, Alexandria University, Egypt \\ Submission: February 27, 2018; Published: March 12, 2018 \\ "Correspondence Address: Nahla AM Hamed, Professor of Hematology, Faculty of Medicine, Alexandria University, Egypt, \\ Email: drhamedn@hotmail.com
}

Abstract

The Hh pathway is a major regulator of many fundamental processes in vertebrate embryonic development including stem cell maintenance, cell differentiation, tissue polarity and cell proliferation. The Hh signaling pathway is activated in many cancers. It has been suggested that $\mathrm{Hh}$ promote cancer by directly regulating cellular growth and/ or survival. Hh signaling also desensitizes tumor cells to radiotherapy and to therapies. Preliminary evidence implicates Hh signaling in diminished immune functions against tumors. Understanding the specific mechanism of Hh activation in a particular tumor might help in optimizing the most appropriate agent and strategy obtained by Hh pathway inhibition.

Abbreviations: Hh: Hedgehog; PTCH: Patched; SMO: Smoothened; Ci: Cubitus Interruptus; JAKi: Janus Kinase Inhibitor; OS: Overall Survival; LDAC: Low Dose Ara-C; CSCs: Cancer Stem Cells; EMT: Epithelial Mesenchymal Transition; GLI: Glioma Associated Oncogene Homolog; MF:

Myelofibrosis; BCC: Basal Cell Carcinoma.

\section{Introduction}

\section{The Hh signaling cascade}

Hh ligand binding to the PTCH, transmembrane protein in the target cell, results in the loss of PTCH activity and the consequent activation of SMO, [1] which then activates a signaling cascade resulting in the translocation of GLI transcription factors in the nucleus [2]. In the nucleus, the GLI transcription factors bind DNA and modulate the transcription of a multitude of genes including Fox, Myc, and cyclin D among others [2]. The Hh signal is transmitted via an alteration of the balance between the activator and repressor forms of the Ci/GLI family of zinc-finger transcription factors [1]. The GLI transcription factors exist as three separate zinc-finger proteins (GLI1, GLI2 and GLI3) [1]. GLI3 acts as the main repressor of the pathway in the absence of Hh ligands; whereas, in the presence of Hh ligands, the main activator, GLI2 leads to the activation of GLI1 promoting its translocation into the nucleus where it initiates the transcription of downstream target genes [2].

The Hh pathway is a major regulator of many fundamental processes in vertebrate embryonic development including stem cell maintenance, cell differentiation, tissue polarity and cell proliferation [1]. It is possible that Hh signaling may be altered in human syndromes caused by defects in cilia, including Meckel, Bardet-Biedl and Kartagener syndromes, polycystic kidney disease, and retinal degeneration [3].

\section{Alteration of the hedgehog pathway and cancer}

The Hh signaling pathway is activated in many cancers [4]. Aberrant activation of Hh signaling can cause rhabdomyosarcoma [3]. Deletions in the receptor patched PTCH or activating mutations in SMO have been reported in BCC and medulloblastoma [4]. GLI1 amplifications were found in gliomas [3]. A variety of other human cancers, including brain, gastrointestinal, lung, breast and prostate cancers, also demonstrate inappropriate activation of this pathway [1]. Hh signaling has also been shown to promote tumor metastasis by being actively involved in the EMT. EMT involves transforming polarized epithelial cells into motile mesenchymal cells facilitating invasive growth and ultimately causing metastasis [1].

The Hh signaling pathway is also aberrantly activated in leukemias and MDS [5]. Hh pathway activity in AML is largely independent of SMO. GLI3 is silenced in most AML patients and the GLI3 locus is abnormally methylated [6]. Expression of Hh target gene has been shown to be up regulated in granulocytes isolated from MF patients [5]. The mechanism of pathway activation in most cancers including hematological malignancies remains unknown [6]. It has been suggested that Hh promote cancer by directly regulating cellular growth and/ or survival [3]. A body of evidence supports the role for 


\section{Cancer Therapy \& Oncology International Journal}

Hh signaling in promoting tumor cell metabolism shift aiding in tumor cell progression, invasiveness and therapy resistance [2]. Hh signaling also desensitizes tumor cells to radiotherapy and to therapies. Emerging preliminary evidence implicates $\mathrm{Hh}$ signaling in diminished immune functions against tumors and the promotion of pro-tumorigenic function by T cells [2].

\section{The proposed modes of Hh signaling pathway activation in cancer are:}

The Hh signaling pathway can be activated classically through ligand binding (autocrine, paracrine and reverse paracrine) or non-classically in the absence of ligand as a result of crosstalk between diverse intracellular signaling networks [2]. In legendsindependent cancers such as BCCs and medulloblastomas, inactivating mutations in PTCH or activating mutations in SMO leads to constitutive activation of the Hh pathway even in the absence of the Hh ligand [1]. Multiple mechanisms have been involved in enhancing GLI1 protein activity in the absence of the Hh ligands [2].

Ligand- (or juxtacrine) dependent autocrine cancers, both produce and respond to the Hh ligand leading to tumor growth and survival e.g. in breast cancer, non-small cell lung cancer, and colorectal cancer [1]. Both classically ligand dependent or non-classically in the absence of ligand are GLI dependent [2]. The truncated isoform of GLI1 is found in cancer tissue and promotes transcription of additional target genes that create a pro-tumorigenic environment for tumor cells to expand [2]. In ligand-dependent paracrine cancers, the Hh ligand secreted by tumor cells activates the pathway in the stroma. The stroma in turn feeds back various signals such as IGF, Wnt, VEGF to the tumor tissue leading to its growth or survival [1], this mainly found in pancreatic cancer [2]. In reverse paracrine tumors, Hh secreted from the stroma lead to pathway activation in the tumor cells and up regulation of survival signals [1]. Multiple myeloma and lymphoma display this mode of action [2]. Hh signaling occurs only in the self-renewing CSCs, from the $\mathrm{Hh}$ ligand produced either by the CSCs or by the stroma. CSC will give rise to more Hh pathway dependent CSCs or possibly may differentiate into Hh-pathway negative tumor cells comprising the bulk of the tumor [1].

The Hh signaling pathway is a promising target for therapeutic development

Understanding the specific mechanism of Hh activation in a particular tumor might help in selecting the most appropriate agent and strategy for optimizing the therapeutic benefit obtained by Hh pathway inhibition. Tumors such as BCC or medulloblastoma, which have a constitutive, mutation-driven activation of the Hh pathway, may be best treated with singleagent $\mathrm{Hh}$ inhibitors acting downstream of the activating mutation. Tumors with predominant autocrine or paracrine Hh signaling and CSCs might be more effectively treated with a combination of $\mathrm{Hh}$ antagonists and cytotoxic drugs targeting tumor cells [1].

The first FDA approved Hh inhibitor for clinical use in 2012 is vismodegib, it targets SMO and is used to treat BCC patients with promising results in other cancer types. Erismodegib is another approved SMO antagonist for treating recurrent BCC patients or for those not qualifies for radiation or surgical removal. Currently, there are several Hh inhibitors employed in clinical trials for multiple types of cancer (sonidegib and glasdegib) [2].

\section{Glasdegib and hematological malignancies}

Glasdegib is a potent and selective oral inhibitor of the Hh pathway, acting through SMO inhibition [7]. In preclinical models of MF, inhibitors of the Hh pathway were shown to inhibit growth and self-renewal of bone marrow stem / progenitor cells that differentiate into cells of the myeloid lineage and to reduce splenic fibrosis. Modest clinical activity was shown in phase $1 \mathrm{~b} / 2$ trial of $100 \mathrm{mg}$ once orally glasdegib in some patients $\geq 18$ years with primary/ secondary MF previously-treated with $\geq 1$ JAKi until there was no further clinical benefit. Some toxicities were observed (dysgeusia, muscle spasm, alopecia, decreased appetite, fatigue, increased lipase, decreased weight, nausea, pyrexia, hyperuricemia, anemia, asthenia, constipation, cough, dehydration, prolonged QT, decreased lymphocyte count, myalgia, pain in extremity, thrombocytopenia and upper respiratory tract infection) [5].

Glasdegib showed significantly better OS when combined with low dose ara-C versus low dose ara-C alone in a randomized phase 2 studies of previously untreated AML patients ineligible for intensive chemotherapy [4]. Hh signaling pathway inhibition reduces leukemic stem cells [5]. Activation of Hh signaling pathway promotes cancer stem cell maintenance, contributes to cancer progression and the development of myeloid leukemia stem cell therapeutic resistance [5]. Hh positively regulates the expression of drug transport pumps in stem cells, enabling them to resist uptake of cytotoxic drugs, makes the strategy of using Hh inhibitors to target the CSCs more rational [1].

The addition of glasdegib to LDAC for high-risk MDS improved OS compared with LDAC alone [8]. The improvement was consistent particularly among good/int. risk patients subgroups. Treatment was associated with an acceptable safety profile [8]. Preclinical results indicate that the combination with venetoclax and glasdegib have potential as an important option for controlling the immature MDS cell population It is expected that the combination with venetoclax and glasdegib may become extremely useful therapeutic interventions to obtain the quick clinical efficacy for MDS patients [7].

\section{Conclusion}

Understanding the mechanisms that control Hh pathway activity will enable development of novel therapeutics for 


\section{Cancer Therapy \& Oncology International Journal}

treating the growing number of developing Hh pathway driven malignancies.

\section{References}

1. Gupta S, Takebe N, Lo Russo P (2010) Targeting the Hedgehog pathway in cancer. Ther Adv Med Oncol 2(4): 237-250.

2. Hanna A, Shevde LA. (2016) Hedgehog signaling: modulation of cancer properties and tumor microenvironment. Molecular Cancer 15: 24.

3. Varjosalo M, Taipale J (2008) Hedgehog: functions and mechanisms. Genes Dev 22(18): 2454-2472.

4. Tremblay G, Arondekar B, Chan G, Shor A, Forsythe A, Yun S. (2017) Covariate adjusted indirect treatment comparison (ITC) of glasdegib plus low dose ara-C versus a hypomethylating agent for acute myeloid leukemia patients ineligible for intensive chemotherapy. Blood 130: 5665 .
5. Gerds AT, Tauchi T, Ritchie EK, Deininger MW, HM Jamieson C, et al. Phase I/II trial of glasdegib in heavily pre-treated patients with primary or secondary myelofibrosis. Blood 130: 258.

6. Chaudhry P, Singh M, Triche TJ, Guzman M, Merchant AA (2017) GLI3 repressor determines hedgehog pathway activation and is required for response to SMO antagonist glasdegib in AML. Blood 129(26): 34653475 .

7. Tauchi T, Okabe S, Katagiri S, Tanaka Y, Ohyashiki K (2017) Combining effects of the SMO inhibitor and BCL-2 inhibitor in MDS-derived induced potent stem cells (Ipsc). Blood 130: 1249.

8. Jorge E Cortes, Florian H Heidel, Michael Heuser, Walter Fiedler, B Douglas Smith, et al. (2016) A phase 2 randomized study of low dose ara-C with or without glasdegib (PF-04449913) in untreated patients with acute myeloid leukemia or high-risk myelodysplastic syndrome. Blood 128: 99.

\begin{tabular}{|l|}
\hline \multicolumn{1}{|c|}{ Your next submission with Juniper Publishers } \\
will reach you the below assets \\
- Quality Editorial service \\
- Swift Peer Review \\
- Reprints availability \\
- E-prints Service \\
- Manuscript Podcast for convenient understanding \\
- Global attainment for your research \\
- Manuscript accessibility in different formats \\
( Pdf, E-pub, Full Text, Audio) \\
- Unceasing customer service \\
Track the below URL for one-step submission \\
https://juniperpublishers.com/online-submission.php \\
\hline
\end{tabular}

